two-sample Mendelian randomisation (MR) analysis; an instrumental variable approach more robust to bias from confounding and reverse causation.

Methods Individual level data from UK Biobank ( $N=217$ 013) was used for multivariable analyses and one-sample MR. Summary statistics from genome-wide association studies were used in two-sample MR.

The total effect of education on risk of coronary heart disease (CHD), CVD (all subtypes), myocardial infarction (MI) and stroke (all measured in odds ratio, OR) was assessed using multivariable regression and univariable Mendelian randomization (MR).

The degree to which this effect is mediated through BMI, SBP and smoking respectively (the indirect effect and proportion mediated) was estimated using the product of coefficients method, where the effect of education on each mediator, and each mediator on each outcome was assessed using multivariable regression and Network MR. The joint contribution of all three risk factors was assessed via the difference method, using multivariable regression or multivariable MR.

Results Each additional standard deviation of education (3.6 years) associated with $13 \%$ lower risk of coronary heart disease (OR $0.87,95 \%$ confidence interval [CI] 0.84 to 0.89 ) in observational analysis and 37\% lower risk (OR 0.63, 95\% CI 0.60 to 0.67$)$ in MR analysis. As a proportion of the total risk reduction, BMI mediated $15 \%$ (95\% CI $13 \%$ to $17 \%$ ) and $18 \%(95 \%$ CI $14 \%$ to $23 \%)$ in the observational and MR estimates respectively. Corresponding estimates for SBP were $11 \%$ (95\% CI $9 \%$ to $13 \%$ ) and $21 \%$ (95\% CI $15 \%$ to $27 \%)$, and for smoking, $19 \%(15 \%$ to $22 \%)$ and $34 \%(95 \%$ CI $17 \%$ to $50 \%$ ). All three risk factors combined mediated $42 \%$ (95\% CI $36 \%$ to $48 \%$ ) and $36 \%$ (95\% CI $16 \%$ to $63 \%$ ) of the effect of education on CHD in observational and MR respectively. Similar results were obtained for risk of stroke, MI and all-cause CVD.

Conclusion BMI, SBP and smoking mediate a substantial proportion of the protective effect of education on risk of cardiovascular outcomes. Intervening on these would reduce cases of CVD attributable to lower education. However, more than half of the protective effect of education remains unexplained.

\section{OP19 WILL SOCIAL CARE NEED MORE RESOURCES? A MODELLING STUDY OF HEALTH AND SOCIAL COSTS IN ENGLAND AND WALES FOR ALTERNATIVE FUTURE CARDIOVASCULAR DISEASE SCENARIOS}

${ }^{1}$ B Collins, ${ }^{1,2}$ P Bandosz, ${ }^{1} \mathrm{M}$ Guzman-Castillo, ${ }^{1,3} \mathrm{~J}$ Pearson-Stuttard, ${ }^{4} \mathrm{G}$ Stoye, ${ }^{5} \mathrm{~J}$ McCauley, ${ }^{3,6}$ S Ahmadi-Abhari, ${ }^{6} \mathrm{MJ}$ Shipley, ${ }^{1} \mathrm{~S}$ Capewell, ${ }^{4,5}$ E French, ${ }^{6}$ EJ Brunner, ${ }^{1} \mathrm{M}$ O'Flaherty*. ${ }^{1}$ Department of Public Health and Policy, University of Liverpool, Liverpool, UK; ${ }^{2}$ Faculty of Medicine, Medical University of Gdansk, Gdansk, Poland; ${ }^{3}$ School of Public Health, Imperial College London, London, UK; ${ }^{4}$ Institute for Fiscal Studies, London, UK; ${ }^{5}$ Department of Economics, University College London, London, UK; ${ }^{6}$ Department of Epidemiology and Public Health, University College London, London, UK

\subsection{6/jech-2019-SSMabstracts. 19}

Background Cardiovascular disease (CVD) contributes to dementia and disability risk. It also affects the cost of care. The English NHS long-term plan targets preventing 150,000 CVD events from 2019-2029. However, after decades of declines in CVD mortality in England, CVD mortality improvements have slowed since 2011, which may indicate a slowdown in incidence reduction from around 2006. Therefore, there is uncertainty about how CVD burden and associated health and social care costs might evolve in the next decade.

Methods Simulations for people aged 35-100 in England and Wales were carried out using the IMPACT Better Aging Model (BAM), an open-cohort, stochastic Markov model which synthesises observed trends in CVD incidence and mortality, dementia and disability in the English Longitudinal Study of Ageing (ELSA) and national ONS data. The synthesised trends were projected to 2029.

We modelled undiscounted health and social care costs and quality adjusted life years (QALYs) for 2019-2029 under two scenarios:

1. Basecase - age-specific CVD incidence continue to decline, following the long-term trends;

2. Age-specific CVD incidence do not decline after 2006, following recent trends.

Healthcare costs were based on hospital episode statistics (HES) data, matched to ELSA participants and calibrated to Office for Budget Responsibility healthcare cost estimates. Age-related social care costs were estimated using reported social care contact hours from ELSA combined with PSSRU unit costs. Utility weights for QALYs were from EQ-5D MEPS catalogue and Health Survey for England.

Results In the basecase scenario 1, median healthcare costs (2019 prices) are projected to increase by $\sim 12 \%$ between 2019-2029, from $£ 93.0$ bn to $£ 104$.6bn per year. Social care costs are projected to increase by $\sim 27 \%$, from $£ 8.0 \mathrm{bn}$ to $£ 10.2$ bn per year.

In the CVD flat-lining scenario 2, median healthcare costs increased by $\sim 15 \%$ from $£ 95.3 \mathrm{bn}$ in 2019 to $£ 109.6 \mathrm{bn}$ in 2029 , and social care costs increased by $\sim 30 \%$ from $£ 8.2$ bn in 2019 to $£ 10.7 \mathrm{bn}$ in 2029 .

When compared with scenario 2, the basecase scenario would generate $\sim 200,000$ additional QALYs/year by 2029, which, valued at UK Treasury rate, would be worth some $£ 12$ billion per year.

Conclusion This study projects future health and social care costs resulting from the recent slowdown in CVD incidence and mortality declines. We predict that social care costs will grow twice as fast as healthcare costs over the next decade, regardless of future improvements. Total funding policy therefore need to needs to be urgently addressed, which may prove politically challenging.

\section{OP20 CARDIOVASCULAR DISEASE BIOMARKER PROFILES AMONG HARMFUL AND HAZARDOUS DRINKERS: A CROSS-SECTIONAL STUDY FROM NORTH WEST RUSSIA}

${ }^{1} \mathrm{O}$ lakunchykova*, 1,2 M Averina, ${ }^{3} \mathrm{~A}$ Kudryavtsev, ${ }^{3} \mathrm{~A}$ Soloviev, ${ }^{1} \mathrm{~T}$ Wilsgaard, ${ }^{1,4} \mathrm{DA}$ Leon. ${ }^{1}$ Department of Comunity Medicine, The Arctic University of Norway University in Tromso, Tromso, Norway; ${ }^{2}$ Department of Laboratory Medicine, University Hospital of North Norway, Tromso, Norway; ${ }^{3}$ Department of Innovative Programs, Northern State Medical University, Arkhangelsk, Russian Federation; ${ }^{4}$ Department of Non-communicable Diseases Epidemiology, London School of Hygiene and Tropical Medicine, London, UK

\subsection{6/jech-2019-SSMabstracts.20}

Background Heavy alcohol drinking is increasingly recognized as a risk factor for cardiovascular disease (CVD), although the mechanisms underlying this are not well understood. Moderate alcohol consumption is associated with changes in many blood biomarkers of cardiometabolic risk. There are however few studies of the impact of harmful and hazardous drinking on 